\title{
Primary Breast Mucormycosis in Immunocompetent Patient - A Rare Case Report
}

\author{
Mahesh H. Karigoudar ${ }^{1 *}$, Rashmi M. Karigoudar ${ }^{2}$, Anirudha V Kushtagi ${ }^{3}$, Ravikumar K Choudhari ${ }^{4}$ \\ ${ }^{1}$ Professor, Department of Pathology BLDEU's Shri B M Patil Medical College Vijayapur, Karnataka State, India \\ ${ }^{2}$ Assistant Professor, Department of Microbiology BLDEU's Shri B M Patil Medical College Vijayapur, Karnataka State, India \\ ${ }^{3}$ Professor, Department of Pathology Koppal Institute of Medical Science Koppal, Karnataka State, India \\ ${ }^{4}$ Professor, Department of Surgery Al-Ameen Medical College Vijayapur, Karnataka State, India
}

DOI: $10.36347 /$ sjmcr.2020.v08i05.012

| Received: 07.05.2020 | Accepted: 14.05.2020 | Published: 19.05.2020

*Corresponding author: Mahesh H. Karigoudar

\section{Abstract}

Mucormycosis is a rare and emerging fungal infection, usually seen in immuno-compromised but can rarely affect immunocompetent persons. Uncontrolled diabetes is a well-known predisposing factor for mucormycosis. The most common reported sites of invasive mucormycosis are nasal sinuses, lungs, and skin. Fungal infections of the breast are uncommon even in patients with immunocompromised status. To the best of our knowledge, less than 10 cases of mammary mucormycosis have been reported till date in the literatures. This fungus invades the blood vessels leading to thrombosis that subsequently causes tissue necrosis and systemic dissemination with high mortality rate. Diagnosis of mucormycosis usually requires culture methods or histopathology. We report a rare case of 40 year old immunocompetent and previously healthy female patient who presented with soft painful mass in the right breast, cracked nipple and areola with the history of application of locally made medicinal paste to the lesion three weeks before consultation. She underwent simple mastectomy and histopathological examination revealed fungal hyphae confirming the diagnosis of Mucormycosis.

Keywords: Mucormycosis, breast, immunocompetent.

Copyright @ 2020: This is an open-access article distributed under the terms of the Creative Commons Attribution license which permits unrestricted use, distribution, and reproduction in any medium for non-commercial use (NonCommercial, or CC-BY-NC) provided the original author and source are credited.

\section{INTRODUCTION}

Mucormycosis is the common name given to several different diseases caused by fungi of the order Mucorales[1, 2]. It is a rare opportunistic fungal infection which was previously known as Zygomycosis. This infection is typically seen in immunocompromised but can occur in immuno-competant person also[2]. Uncontrolled diabetes is a well known predisposing factor for mucormycosis[2, 3]. The most common reported sites of invasive mucormycosis are nasal sinuses (39\%), lungs (24\%), and skin (19\%)[4]. Fungal infections of the breast are uncommon even in patients with immunocompromised status[5]. To the best of our knowledge, less than 10 cases of mammary mucormycosis have been reported till date in the literatures. This fungus invades the blood vessels leading to thrombosis that subsequently causes tissue necrosis and systemic dissemination with high mortality rate[1-3].

Disease presentation differs in terms of organs affected and also depends on insidious or rapid course. The varied clinical manifestations and vague presentation of mucormycosis often delay diagnosis with consequential poor outcomes. Diagnosis of mammary mucormycosis may be difficult only on clinical features and it requires culture methods and histopathological examination [4]. It can be treated by systemic antifungal agents with surgical debridement or simple mastectomy. Here we report a rare case of breast mucormycosis in immunocompetent and nondiabetic woman.

\section{CASe Report}

A 40 year old healthy female presented with soft painful mass in the right breast, cracked nipple and areola since 15 days. There was a history of application of locally prepared medicinal paste to the nipple and areola three weeks before consultation. Pain was increased in intensity since 3 days before consultation. On examination the breast mass was soft, swollen, tender and warm. Nipple and areola was red and cracked. Hematological investigations were within normal limits. She was non diabetic and non-reactive for HIV antibodies and no signs of other chronic illness. Fine needle aspiration cytology of the breast mass was 
performed and it was inconclusive. Because of intense pain and soft mass in the breast, clinicians decided for surgical intervention and patient underwent simple mastectomy.

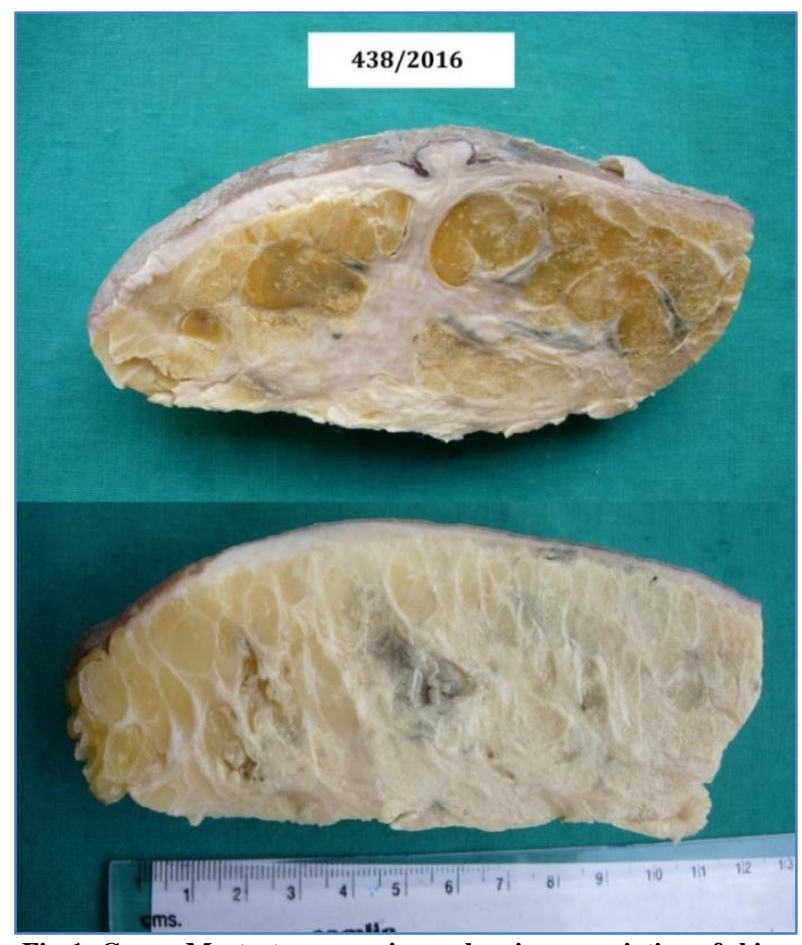

Fig-1: Gross: Mastectomy specimen showing excoriation of skin. Cut surface shows pale yellow with extensive pale grey-white necrosis

Examination of mastectomy specimen measuring $13 \times 10 \times 6 \mathrm{~cm}$ and revealed excoriation of the skin and cut section showed yellowish and pale greywhite necrotic areas (figure 1). Microscopic examination of haematoxylin and eosin stained sections revealed areas of necrosis (figure 2) which had broad non-septate irregular fungal hyphae with obtuse branching (figure 3,4 ), foci of vascular invasion by the fungi and mixed inflammatory cell infiltration (figure 5). The fungi were positive for periodic acid Schiff stain confirming the diagnosis of Mucormycosis of breast.

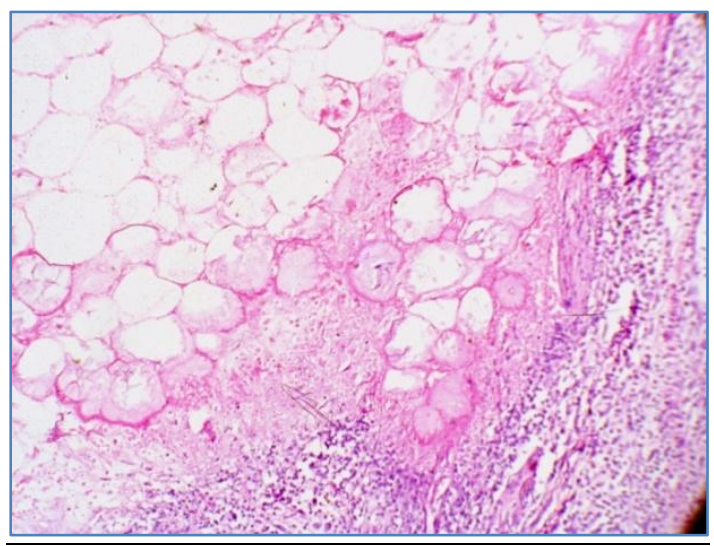

Fig-2: Microphotograph showing extensive fat necrosis with inflammatory cell infiltrate $(\mathrm{H} \& \mathrm{E}, 100 \mathrm{X})$

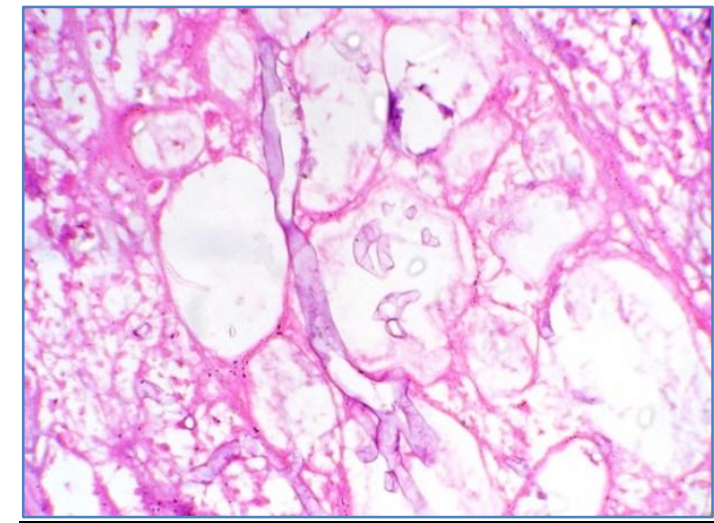

Fig-3: Microphotograph showing adipose tissue with broad nonseptate irregular hypae of mucormycosis $(\mathrm{H \& E}, 400 \mathrm{X})$

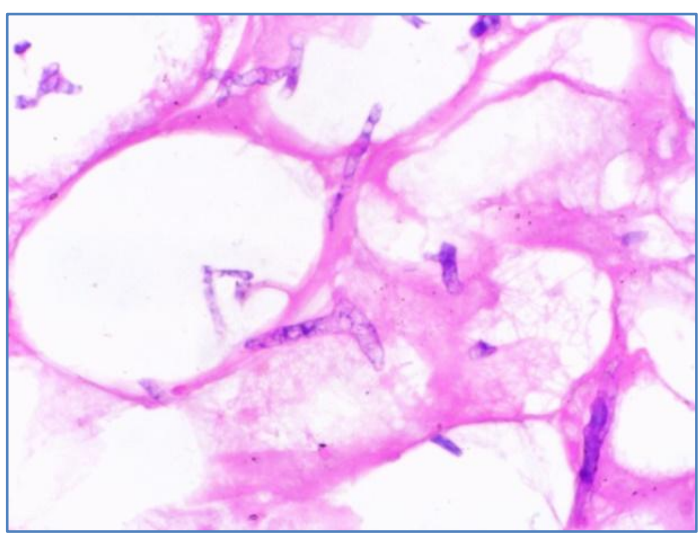

Fig-4: Microphotograph showing adipose tissue and obtuse branching of mucormycosis hypae (H\&E, 400X).

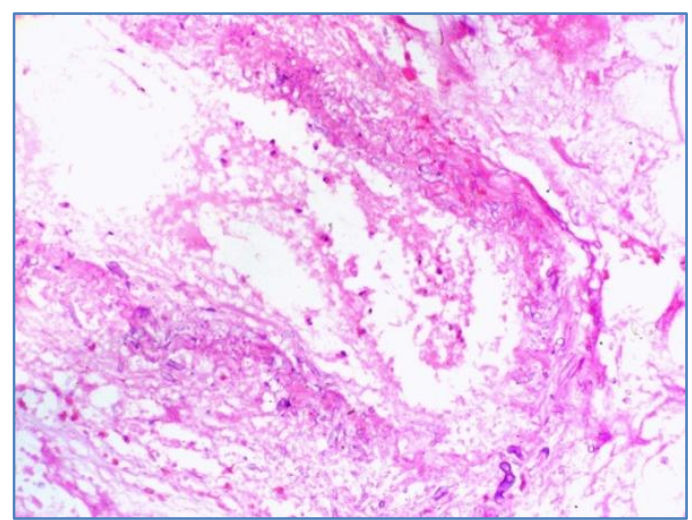

Fig-5: Microphotograph showing mucormycosis invading the vacular wall $(\mathrm{H} \& \mathrm{E}, 100 \mathrm{X})$

Patient responded well with regular postoperative open dressings of the wound with i.v administration of amphotericin. Wound healed completely with skin graft and she was free from complications upto two years of follow up.

\section{DISCUSSION}

Mucormycosis is the common name given to several diseases caused by fungi in the order of Mucorales[1-3]. It is an acute opportunistic infection caused by a saprophytic fungus that belongs to the class of phycomycetes[2]. Although several genera are associated with this disease, the most common forms 
are Rhizopus, Rhizomucor, Cunninghamella and Absida[1,2]. They are saphrophytic and low virulent fungi usually found in the soil, spoiled foods and air dust. Even though most individuals are exposed to these fungi but, can cause fatal infection only in certain immuno compromised conditions ${ }^{3}$. Predisposing factors include poorly controlled diabetes mellitus with ketoacidosis facilitates dissemination of the infection, malignant patients on chemotherapy and radiotherapy, chronic illness like cirrhosis of liver and renal failure, conditions like: burns, protein energy malnutrition, neutropenia and acquired immune deficiency syndrome, organ transplant, long term corticosteroid and immunosuppressive therapy[2,3]. Functional defects of neutrophils and macrophages in diabetics and steroidtreated patients may facilitate the opportunistic fungal infections like mucormycosis and its disseminations [2]. To begin with, cutaneous lesions are erythematous to purple painful indurated plaques. These develop into eschar due to erythematous halo and necrosis. Rarely these lesions present with targetoid lesions, painful nodules, ulcers, purpuric lesions, and swollen mass and scaly plaques. In surgical wound infections and burns patients, mucormycosis appears as cellulitis and necrosis [6].

In the present case, patient was not having any underlying predisposing factor for mucormycosis, patient was non diabetic and her serological test for HIV antibodies was non-reactive. The cause of susceptibility for the infection was unknown and possibly the fungi might have gained entry through the injured skin over breast, as patient had history of application of in-house prepared paste for her cracked nipple and areola.

Mucormycosis is one of the most rapidly progressing and lethal form of fungal infection in humans ${ }^{2}$. The clinical patterns of the disease produced by different genera or species of Mucorales are virtually identical. Mucormycosis usually presents as an acute infection and may present as: rhinocerebral, pulmonary, cutaneous, gastrointestinal, central nervous system and miscellaneous lesions. Less than 10 cases of primary breast mucormycosis have been reported in the literature till date. Clinically apparent fungal infections of the breast are uncommon, even in patients who are severely immuno-compromised [7].

A definitive diagnosis of mucormycosis can be made by tissue biopsy that identifies the typical hyphae, by positive culture growth or both. Initial culture of diseased tissue will longer time and may be negative and hence histopathological examination is essential for early diagnosis, the fungus can be further confirmed by special stains like Periodic acid Schiff and Grocott's silver methenamine[1,2]. The classical microscopic features include necrotic and edematous tissue, neutrophilic infiltration and presence of broad, nonseptate hyphae with obtuse branching with vascular invasion [1-3]. All these finding were also consistent in the present case, Periodic acid Schiff stain highlighted the fungal hypae confirming the diagnosis of Mucormycosis. Fungal culture was not done probably clinically fungal infection was not suspected.

Mucormycosis was long regarded as a fatal infection with poor prognosis. However with early medical and surgical management survival rates are now thought to exceed $80 \%$. Measures like controlling the underlying predisposing factors for such infections are also important for its prevention and control of further dissemination [8]. The present case was treated by simple mastectomy with open dressings and administration of systemic amphotericin. The patient was in good health without any complications upto follow up of two years.

\section{CONCLUSION}

Fungal infections of the breast are uncommon even in patients with immuno-compromised status. Primarily Mucormycosis affects immuno-compromised but can occur in healthy or immunocompetent persons also. Demonstration of fungi on histopathological examination is essential for its early diagnosis. Though it is fatal infection, the outcome can be improved by early diagnosis, surgical intervention, and regular wound dressings after surgery and systemic antifungal drugs like amphotericin B.

\section{REFERENCES}

1. Thapar VK, Deshpande A, Jain VK, Bhowate P, Madiwale C. Isolated breast mucormycosis. J Postgrad Med. 2006; 52:134-5.

2. Auluck A. Maxillary necrosis by mucormycosis. A case report and literature review. Med Oral Pathol Oral Cir Bucal. 2007; 12:E360-4.

3. Baezzat SR, Fazelzadeh A, Tahmasebi S, Kumar PV. Primary Breast Mucormycosis, A Case Report. Iran Red Crescent Med J. 2011; 13(3): 208-209.

4. Kataria SP, Sharma J, Singh G, Kumar S, Malik S, Kumar V. Primary breast mucormycosis: FNAC diagnosis of a rare entity. Diagn Cytopathol. 2016;44(9):761-763

5. Sukhamwang N, Chaiwun B, Trakultivakorn H. Fungal infection of the breast: Two reported cases diagnosed by Fine needle aspiratation (FNA) cytology. Chiang Mai Med J. 2009;48(2):65-70.

6. Castrejón-Pérez AD, Welsh EC, Miranda I, Ocampo-Candiani J, Welsh O. Cutaneous mucormycosis. An Bras Dermatol. 2017 MayJun;92(3):304-311

7. McNulty JS. Rhinocerebral mucormycosis: predisposing factors. Laryngoscope. 1982;92:11403.

8. Muma MKI, Mutati GC, Deadly Orbital Mucormycosis, Rare yet Possible Infection. Medical Journal of Zambia.2010; 37(4): 268-72. 